\title{
El Aprendizaje Basado en Problemas: Una herramienta didáctica para el aprendizaje significativo de las ciencias químicas en la Universidad Alas Peruanas
}

\section{Problem-based Learning: A didactic tool for the significant learning of chemical sciences at the Alas Peruanas University}

\author{
Miriam Vilca ${ }^{1}$
}

\section{RESUMEN}

El objetivo de la investigación es el análisis del impacto de la integración del Aprendizaje Basado en Problemas (APB ) en los estudiantes como estrategia didáctica para la enseñanza del curso de Química. La muestra poblacional se compone de estudiantes del tercer ciclo del pregrado, de la Facultad de Ingeniería Industrial y Civil de la Universidad Alas Peruanas (UAP). Se utilizó una metodología cuantitativa correlativa, bajo un muestreo probabilístico, utilizando encuesta y lista de cotejo.

Los resultados fueron: relación significativa entre la integración del aprendizaje ABP como enseñanza del curso del curso Química en los estudiantes del tercer ciclo de la Facultad de Ingeniería Industrial y Civil de la Universidad Alas peruanas tiene una correlación alta $(0,776)$ y con una significancia $(0.02)$. El ABP se relaciona con la enseñanza del curso de Química, el cual presenta una correlación directa de 72.0\% y la aplicación del ABP se relaciona con la enseñanza del curso de Química, el cual presenta una correlación directa de $81.0 \%$.

Palabras clave: ABP, didáctica, aprendizaje significativo, enseñanza.

\section{ABSTRACT}

The objective was to analyze the impact of the students to integrate the BPL, as a didactic strategy in the teaching of the course of Chemistry with the students of the third cycle of the Faculty of Industrial and Civil Engineering of the University Alas Peruanas. A correlative quantitative methodology was used, under a probabilistic sampling, using survey and checklist.

The results were: significant relation between the integration of BPL as teaching of the chemistry course in students of the third cycle of the Faculty of Industrial and Civil Engineering of the Peruvian Alas University has a high correlation (0.776) and with a significance (0.02). The BPA is related to the teaching of the Chemistry course, which has a direct correlation of $72.0 \%$ and the application of BPL is related to the teaching of the Chemistry course, which has a direct correlation of $81.0 \%$

Keywords: ABP, didactics, meaningful learning, teaching.

1. Ingeniero Químico. Universidad Alas Peruanas - Filial Chincha. Email:mc.ing.miriamvilca@gmail.com; http://orcid.org/0000-00024898-4569. 


\section{INTRODUCCIÓN}

La complejidad del proceso educativo es un desafío aceptado y estudiado por expertos pedagogos, operadores de la educación, científicos sociales y la sociedad misma, desde diferentes perspectivas, como parte de la formación de ciudadanos capaces de afrontar nuevas formas de vida en un mundo altamente competitivo.

Más allá, en la sociedad del conocimiento, se da su inserción en la sociedad de la economía del conocimiento y la innovación.

El logro del aprendizaje de una materia o disciplina, se basa en la manera de cómo se trasmite los conocimientos.

Esa manera es la didáctica que prefiguró magistralmente Comenio hace más de tres siglos, la misma que hoy cobra importancia trascendental. Aprender para aprender, aprender a desaprender, como se sugiere en los pilares de la educación planteada por la Unesco, y que sirve de marco de fondo para los estudios científicos en los claustros universitarios a nivel mundial.

Bajo esta perspectiva, el presente artículo centra sus estudios en el impacto del APB , como un método didáctico centrado en el rol protagónico del estudiante en contraposición a los métodos tradicionales, favoreciendo dramáticamente el aprendizaje significativo reclamado por David Ausubel.

El estudio analiza de manera crítica la aplicación del método en el proceso de enseñanza y aprendizaje de la materia química en estudiantes del tercer ciclo las carreras profesionales de ingeniería Industrial y Civil de la UAP y en las demás universidades peruanas, con acento tradicional. Esto debido a que los docentes en su mayoría provienen de formaciones profesionales que no manejan técnicas provenientes de la pedagogía, la misma que nos permitió trazar el objetivo de analizar detalladamente el impacto de esta estrategia y fomentar la aplicación didáctica en las universidades para lograr aprendizajes significativos, como una novedosa propuesta pedagógica.

El ABP es importante porque permite al estudiante desarrollar el ejercicio de razonar amplia e integralmente logrando un aprendizaje de "aprender para aprender". El estudiante desarrolla su capacidad resolutiva para diversas problemáticas planteadas en el curso, logrando un pensamiento complejo y de actitud crítica.

La pedagogía tradicional busca esencialmente la formación del pensamiento empírico, donde el estudiante, a través del aprendizaje, es un receptor pasivo, mientras que el docente al realizar la enseñar, se considera como un emisor (i) activo. El conocimiento se asimila por aproximaciones sucesivas, se ofrece como verdades acabadas $y$, generalmente existe un insuficiente vínculo con la vida.

Es imprescindible unificar los esfuerzos de los educadores entorno a la generación y aplicación de métodos y procedimientos más generales y productivos que complementen los diferentes métodos que, de forma coherente, integren la acción de las diversas áreas del conocimiento que influyen sobre el estudiante, con el fin de lograr una mayor participación colectiva y consciente para el desarrollo de su creatividad, su pensamiento e imaginación.

El ABP es sumamente importante para que el estudiante pueda razonar de manera generalizada y concretar un aprendizaje hecho para aprender a aprender. Siendo quien resuelve los problemas a través del razonamiento, logrando de esta manera un pensamiento complejo y una actitud crítica en el joven.

A nivel nacional, la Pontificia Universidad Católica del Perú (PUCP), conjuntamente con la Red Panamericana para el Aprendizaje Basado en Problemas, organizaron el Congreso Internacional de ABP 2006 , con el objetivo de generar un espacio para la presentación y discusión de investigaciones, experiencias y materiales relativos 
a la implementación del ABP (1). En este evento se desarrollaron diversas actividades como talleres, conferencias a cargo de expositores nacionales como internacionales, presentación de experiencias e investigaciones sobre la aplicación e implementación del ABP en distintos países del mundo.

A nivel regional, la metodología APB no es aplicada ni reconocida entre los profesionales que ejercen la enseñanza de diversas materias a nivel universitario.

Sin duda alguna, la solución de problemas como contenido educativo tiene un carácter esencialmente procedimental, ya que, requiere que los alumnos pongan en marcha una secuencia de pasos de acuerdo con un plan preconcebido y dirigido al logro de una meta (2).

Aprender a través de la comprensión, la problematización y la toma decisiones, facilita el aprendizaje significativo porque promueve que los estudiantes establezcan relaciones significativas entre lo que ya saben y la nueva información, y que ello perdure en niveles más profundos de apropiación.

A través de las distintas experiencias pedagógicas cursadas de la enseñanza del curso de química se puede observar que los estudiantes demuestran poco interés y poco compromiso con la materia, lo cual obstaculiza el sentido del aprendizaje significativo y comprensivo, que deriva en un aprendizaje pobre y casi mecánico, memorístico, escasamente transferible de los contenidos (3).

Sandoval et al. (4), afirma: Esta situación nos impone el reto de buscar, construir y aplicar alternativas educativas que generen interés, curiosidad y gusto por aprender, dialogando con los docentes de otras asignaturas básicas correspondientes a los primeros años de la carrera, estos comentan que perciben el mismo problema.

De esta manera podemos avizorar que, es de vital importancia para nuestro sistema educativo, la integración de la nueva modalidad que nos brinda la estrategia de aprendizaje ABP.

\section{Cuyos objetivos de estudios específicos son:}

a) Indagar en los estudiantes del III ciclo de la Facultad de Ingeniería Industrial y Civil, las opiniones respecto de la adquisición de competencias a través del Aprendizaje Basado en Problemas, como estrategia didáctica para la enseñanza del curso de Química.

b) Conocer los niveles de relación entre el ABP y la enseñanza del curso de Química.

c) Identificar y explicar los factores de carácter pedagógico-didáctico condicionantes del nivel de rendimiento académico en el curso de química detectado en los estudiantes.

d) Describir los niveles de eficiencia del $\mathrm{ABP}$ aplicado a la enseñanza del curso de Química.

\section{MATERIALES Y MÉTODOS}

El diseño de esta investigación es no experimental, es decir, es aquella que se realiza sin manipular deliberadamente variables. Es decir, es la investigación en donde no se hace variar intencionalmente las variables independientes.

El diseño de la investigación es descriptivo con el fin de lograr una visión clara del problema describiendo una analizando el fenómeno de la deserción a través de la medición independiente de variables estableciendo relaciones de causa efecto.

Además comprende varios niveles de análisis, a partir de un diseño no experimental transaccional exploratorio, cuyo propósito es analizar los factores determinantes de la deserción estudiantil. 


\section{RESULTADOS}

\section{Gráfico $\mathrm{N}^{\circ} 1 \mathrm{ABP}$ integrado a la clase de Química}

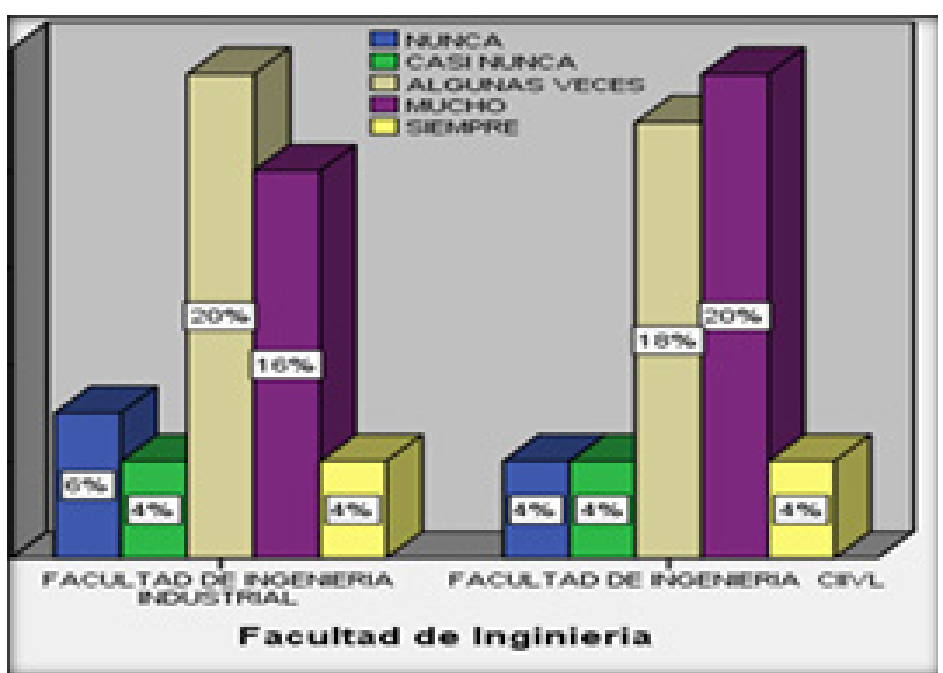

El ABP integrado a la clase de Química brinda aportes significativos a la clase.

\section{Gráfico $\mathrm{N}^{\circ} 2 \mathrm{ABP}$ Estrategias experimentales}

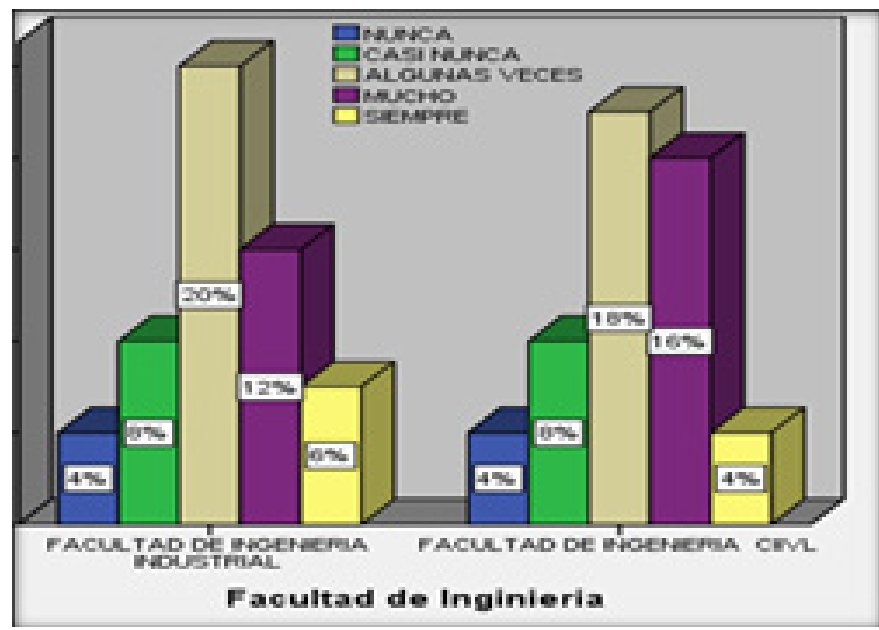

Las estrategias experimentales del $\mathrm{ABP}$, en la práctica de laboratorio mejoraron la enseñanza y aprendizaje de la clase de química. 


\section{Gráfico $\mathrm{N}^{\circ} 3 \mathrm{ABP}$ Información analizada}

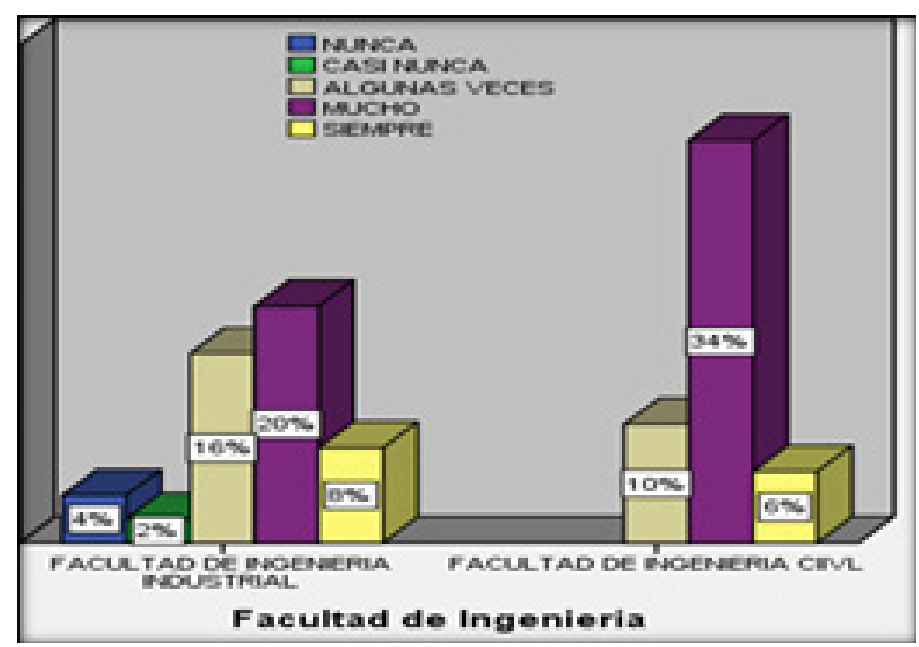

Relación de la información analizada en las sesiones del ABP.

\section{DISCUSIÓN}

Los resultados obtenidos a través de la descripción de las variables y las Pruebas de Hipótesis nos permiten sustentar e interpretar en forma adecuado, precisa y completa nuestros resultados lo cual nos permite afirmar respecto a una relación significativa entre la integración del aprendizaje ABP como enseñanza y el aprendizaje de la química en los estudiantes del III ciclo de la Facultad de Ingeniería Industrial y Civil de la Universidad Alas Peruanas, con una correlación alta $(0,776)$ y con una significancia $(0.02)$.

Con Respecto a la opinión de los estudiantes del III ciclo de la Facultad de Ingeniería Industrial y Civil, opinan favorablemente respecto del Aprendizaje Basado en Problemas(ABP) como estrategia para la adquisición de competencias elementales en la enseñanza y aprendizaje de la Química, hay una relación significativa, con una correlación alta $(0,751)$ y con una significancia (0.03), resulta indicar en relación a la opinión los que el Aprendizaje Basado en Problemas está íntimamente relacionado a la enseñanza y aprendizaje de la Química, hay una relación significativa, con una correlación alta $(0,720)$ y con una significancia (0.01), los factores de carácter pedagógico-didáctico condicionantes del rendimiento académico de los estudiantes, en la enseñanza y aprendizaje de la Química, se evidencia una relación significativa, con una correlación alta $(0,710)$ y con una significancia $(0.01)$, respecto a la aplicación del aprendizaje basado en problemas mejora la enseñanza y aprendizaje de la Química, se evidencia una relación significativa con una correlación alta $(0,810)$ y con una significancia (0.02).

El 38\% de los estudiantes de la Facultad de Ingeniería Civil, percibe que se realiza motivaciones para atraer la atención y solo $32 \%$ estudiantes de la Facultad de Ingeniería Industrial percibe que se realiza motivaciones para atraer la atención, lo evidencia la poca factibilidad por parte de los docentes por atraer la atención de los estudiantes de ambas facultades de Ingeniería de la Universidad Alas Peruanas.

Considerar que solo el $20 \%$ de los estudiantes de la Facultad de Ingeniería Industrial considera que algunas veces al integrar el ABP a la clase de química brinda aportes significativos a la clase, el $20 \%$ de los estudiantes de la Facultad de Ingeniería Civil considera que contribuye mucho integrar el $\mathrm{ABP}$ a la clase de química brindando aportes significativos a la clase, lo cual se puede afirmar que el $80 \%$ de estudiantes de ambas fa- 
cultades de Ingeniería de la Universidad Alas Peruanas, desconoce la importancia del aprendizaje del ABP, lo cual habría que dar un impulso en la Universidad Alas Peruanas.

Recalcar que el $20 \%$ de los estudiantes de la Facultad de Ingeniería Industrial considera que han mejorado mucho sus habilidades en las prácticas de laboratorio durante la enseñanza de la química, el 20 \% de los estudiantes de la Facultad de Ingeniería Civil considera que han mejorado mucho sus habilidades en las prácticas de laboratorio durante la enseñanza de la química, del cual se debe dar esfuerzo en poder brindar e implementar del aprendizaje del ABP para la mejora de sus conocimientos, lo cual se puede constatar sobre la opinión de sesiones del ABP como se indica el resultado de la encuesta que solo el 20 $\%$ de los estudiantes de la Facultad de Ingeniería Industrial considera haber logrado mucha en concordancia con la información analizada en las sesiones de ABP, el $34 \%$ de los estudiantes de la Facultad de Ingeniería Civil considera haber logrado mucha en concordancia con la información analizada en las sesiones de ABP, lo que infiere todo lo mencionados por los diferentes autores respecto a la importancia de llevar de implementar y llevar acabo el aprendizaje del ABP, en la Universidad Alas Peruanas.

\section{CONCLUSIONES}

El presente estudio consideramos que el Aprendizaje Basado en Problemas como estrategia didáctica se relaciona con la enseñanza y aprendizaje de la Química en los estudiantes del III ciclo de la Facultad de Ingeniería Industrial y Civil de la Universidad Alas Peruanas, el cual presenta una correlación directa de $77.6 \%$.

Los estudiantes del III ciclo de la Facultad de Ingeniería Industrial y Civil, hay una relación con respecto al Aprendizaje Basado en Problemas como estrategia para la adquisición de competencias elementales en la enseñanza y aprendizaje de la Química, el cual presenta una correlación directa de $75.1 \%$.
El Aprendizaje Basado en Problemas se relacionada con la enseñanza y aprendizaje de la Química, el cual presenta una correlación directa de $72.0 \%$.

Los factores de carácter pedagógico-didáctico se relacionan con el rendimiento académico de los estudiantes, en la enseñanza y aprendizaje de la Química, el cual presenta una correlación directa de $71.0 \%$.

La aplicación del Aprendizaje Basado Problemas se relaciona con la enseñanza y aprendizaje de la Química, el cual presenta una correlación directa de $81.0 \%$.

\section{REFERENCIAS}

1. Méndez Lee J. solución de problemas como contenido Procedimental de la Educación Obligatoria. En Pozo J. et. al.: "La solución de Problemas. Editorial Santillana, Madrid, España. 1994.

2. Pozo J., Del Puy Pérez M. La solución de problemas como contenido Procedimental de la Educación Obligatoria. En POZO J. et. Al.: "La solución de Problemas. Editorial Santillana, Madrid, España. 1994.

3. Sandoval M., Maldonesi M., Cura R.: Estrategias didácticas para la enseñanza de la Química en Educación Superior). Universidad Tecnológica Nacional. Facultad Regional Bahía Blanca. Argentina. 2013

4. Rodríguez Palmero M. La Teoría del Aprendizaje Significativo. Centro de Educación a Distancia (C.E.A.D.). C/ Pedro Suárez Hdez, s/n. C.P. no 38009. Santa Cruz de Tenerife. España). 2004 